Bioscientia Medicina Volume 4, Issue 3, Page No: 10-18 Available online : www.bioscmed.com Bio Sc Med 4(3) :10-18

\title{
Correlation of Lymphocyte-Monocyte Ratio with Grading of Breast Cancer Malignancy
}

\author{
Muhartono $^{1 \#}$, Rizki Hanriko ${ }^{1}$, Suharyani ${ }^{2}$ \\ ${ }^{1}$ Department of Anatomy, Patology Anatomy and Histology, Faculty of Medicine, University of Lampung \\ ${ }^{2}$ Department of Biochemistry, Molecular Biology, and Physiology, Faculty of Medicine, University of Lampung \\ \#Corresponding Author E-mail: muhartono.unila@gmail.com
}

\begin{abstract}
Introduction: Breast cancer cases are increase and fast becoming the leading cause of oncologic mortality among women worldwide. Delay in management will worsen the prognosis of breast cancer patients. The poor prognosis is correlate with high grade of breast cancer malignancy. Histology can be used as an indicator to assess the grade of breast cancer malignancy. In addition, a high predictive value of lymphocyte-monocyte ratio (LMR) has been suggested to indicate favorable prognosis of various cancer, and used as parameters for assessing breast cancer prognosis.
\end{abstract}

Aim of study: The purpose of this study is to assess the correlation between lymphocytemonocyte ratio and the grade of breast cancer malignancy.

Method: This study is a quantitative research, using an observational approach. Laboratory examination results are collected from 43 breast cancer patients who will conduct treatment at Urip Sumoharjo Hospital from June to September 2019. Data are processed by the Spearman rank test using SPSS 22.0.

Results: Correlation test results between lymphocyte-monocyte ratio with histological grading of breast cancer obtained a $\mathrm{P}$ value of 0.376 .

Conclusions: Lymphocytes to monocytes ratio (LMR) value cannot be utilize as a parameter for evaluating prognosis of breast cancer.

Keywords: breast cancer, grade of malignancy, lymphocyte-monocyte ratio (LMR). 
ISSN 25980580

\section{Introduction}

Cancer is the most common disease in the world for the last decades. Breast cancer is the second most common type of cancer after lung cancer. According to data from World Health Organizaton (WHO), 2,1 million new cases of breast cancer were diagnosed in 2018. One in four women who diagnosed with cancer $(24,2 \%)$ worldwide is breast cancer. ${ }^{1}$ The incidence rate of breast cancer occupy the most cancer incidence in 25 countries worldwide, especially in developing Countries. ${ }^{1,2}$ There are 4,933 new cases of breast cancer incidence, increased from 12.023 cases in 2017 to 16.955 cases in 2018. ${ }^{3,4}$ Most cases in Indonesia were occur in the province of West Java (4,141 cases) followed by Lampung in the fourth place with 1.836 cases occur in $2018 .^{4}$

Breast cancer is one of the five biggest leading causes of death in the world. Data from WHO in 2018, shows about 627.000 cases or $6,6 \%$ cancer mortality rate are caused by breast cancer. Estimated about $15 \%$ of total mortality in women are caused by breast cancer, making it the most leading cause of death in women. ${ }^{1}$ In 2018 , the mortality rate is estimated at 42.1 per 100,000 population due to breast cancer in Indonesia and is the highest rate among women. ${ }^{4}$

Breast cancer common diagnosed with a relatively good prognosis. The average 5-year survival rate for women with breast cancer are around $80-90 \%$. The delayed diagnosis is responsible in causing poor prognosis and even mortality of the patient. ${ }^{5}$ The prognosis of breast cancer can be assessed by histopathological gradations according to the Nottingham Score which is divided into three grades, grade I (well differentiated), grade II (moderat differentiated), and grade III (poorly differentiated). The higher grade of Nottingham Score is associated with poor prognosis. ${ }^{6-8}$

However, it is not only the characteristics of the tumor that can determine the prognostic of the cancer. The inflammatory response from the host can also be used as a parameter for determining the prognosis of cancer. ${ }^{9}$ Several studies have shown that systemic inflammation can stimulate tumor development by inhibiting apoptosis, stimulating angiogenesis and DNA damage. Hematologic index for systemic inflammation, such as leukocyte count, monocyte count, platelet count, neutrophil-lymphocyte ratio (NLR), lymphocyte-monocyte ratio (LMR) and platelet-lymphocyte ratio (PLR) have been proposed as prognostic factors in cases of lung, stomach and breast cancer. ${ }^{10}$ 
Several studies have shown that LMR is associated with the prognosis of cancer. ${ }^{11}$ A low preoperative lymphocyte-monocyte ratio is associated with a poor prognosis in patients with cervical cancer and several other types of cancer. ${ }^{12}$ Low lymphocyte-monocyte ratio is associated with poor life expectancy of patients with breast cancer. ${ }^{9}$ According to that, this study aim to evaluate the correlation of the lymphocyte-monocyte ratio with grading of breast cancer malignancy.

\section{Methods}

Quantitative study with observational approach were used in this study. It was conducted from June to September 2019 at three laboratory, which are Clinical Pathology Laboratory of Urip Sumoharjo Hospital, Anatomical Pathology Laboratory of Urip Sumoharjo Hospital and Anatomy, Histology and Phatology Anatomy Laboratory, Faculty of Medicine, University of Lampung. This study used laboratory examination results of breast cancer patients who will conduct treatment at Urip Sumoharjo Hospital Bandar Lampung in June - September 2019 as the data. This study were using consecutive sampling as sample selection method with inclusion criteria as followed; (i) no history of cancer before; (ii) never received chemotherapy treatment; and (iii) not taking anti-inflammatory medication before. Based on these inclusive criteria, 43 samples were obtained in this study.

This study began with the collection of breast cancer patients at Urip Sumoharjo Hospital during the period of June - September 2019 according to patient's medical record. Samples that have fulfilled the criteria are then continue to laboratory examination. There are basic hematology parameter test, and histopathological examination of breast biopsy from each laboratory. Examination data obtained in the form of hemoglobin, leukocytes, neutrophils, lymphocytes, monocytes and platelets count for hematological examination parameters as well as the type of histology and grade of breast cancer malignancy on histopathological examination.

The results of this study are numerical data (ratios) for basic hematological test parameters and categorical data for breast cancer grading. Data were analyzed with the Spearman rank test using the SPSS 22.0 device to evaluate the correlation between lymphocyte-monocyte ratio and the grade of malignancy in breast cancer patients. 
ISSN 25980580

\section{Results}

Examination has been carried out on 43 breast cancer patients who will undergo treatment at Urip Sumahardjo Hospital in June - September 2019 who have met the inclusion criteria. Charatacteristic of patients age and ditribution was divided into four groups with the highest frequency in the 40-50 group with a total of 25 people (58.14\%). The age distribution of the study sample is described in table one.

Data on hematological examination results from 43 samples are described in highest, lowest and average values of all samples. Assessment of lymphocyte-monocyte ratio (LMR) is determine by dividing the value of the lymphocytes count by monocytes count. The highest, lowest and average values of LMR are 10; 0,36; and 3,87 respectively. Characteristics of haematology and LMR examination results are described in table two.

Histopathological examination results of 43 samples were described into two categories, there are classification of breast cancer types and the histological grading. Type of breast cancer are classified into three categories based on specific cells that are affected. Most frequent type is ductal carcinoma invasion, found in 35 samples (81.39\%). While the classification based on the histological grading divided into three grades with the most common is grade III, found in 28 samples (65.12\%). Result of Hisopathological examination characteristic are described in table three.

Correlation test result between grading of malignancy and basic haematological test parameter (Hb, Leukocytes, and platelet count) are described in table four. Table four shows various results for each parameter. Spearman Rank correlation test results between grading of malignancy with Hb levels $(r=0.251 ; p=0.104)$ and platelets $(r=-0.091 ; p=0.562)$, does not show a significant correlation statistically. In line with the previous parameters, grading of malignancy does not show a correlation with the number of leukocytes $(r=0.111, p=0.479)$, with neutrophils $(r=0.100 ; p=0.484)$, with lymphocytes $(r=-0.227 ; p=0.144)$, and with monocytes $(r=-0.017 ; p=0.915)$.

Spearman Rank test results show LMR values $<2.5$ are found in 11 people with grade III (25.58\%), followed by three people with grade II (6.98\%). LMR values range from 2.5 to 5.0 were found in 11 samples with grade III (25.58\%), 6 samples with grade II (13.95\%) and 2 
samples with grade I $(4.65 \%)$. While the LMR value $>5.0$ were found in six people with grade III (13.95\%) and four people with grade II (9.3\%). The results of the Spearman Rank Test between the grading of malignancy and LMR are described in table five.

Table 1. Age Distribution of Samples

\begin{tabular}{ccc}
\hline Age & Frequency & Percentage \\
\hline$<40$ years old & 5 & $11,63 \%$ \\
$40-50$ years old & 25 & $58,14 \%$ \\
$51-60$ years old & 11 & $25,58 \%$ \\
$>60$ years old & 2 & $4,65 \%$ \\
\hline
\end{tabular}

Table 2. Characteristics of Hematology and LMR Examination Results

\begin{tabular}{lccc}
\hline \multicolumn{1}{c}{ Variables } & Min. & Max. & Mean \pm SD \\
\hline Hemoglobin $(\mathrm{g} / \mathrm{dL})$ & 7,1 & 15,6 & $11,7 \pm 1,8$ \\
Lekocyte $(\mathrm{Sel} / \mu \mathrm{L})$ & 1.250 & 16.060 & $7760,69 \pm 2971$ \\
Neutrophil $(\%)$ & 32 & 93 & $63,58 \pm 14,3$ \\
Limphocyte $(\%)$ & 5 & 47,7 & $25,64 \pm 13,57$ \\
Monocyte $(\%)$ & 2 & 41,8 & $9,08 \pm 0,8$ \\
Platelet & 91.000 & 515.000 & $276.000 \pm 89.583$ \\
LMR & 0,36 & 10 & $3,87 \pm 3,06$ \\
\hline
\end{tabular}

Table 3. Characteristics of Histopathological Examination Results

\begin{tabular}{lcc}
\multicolumn{1}{c}{ Variables } & Frequency & Percentage \\
\hline Breast cancer type & & \\
-. Invasif Ductal Carcinoma & 35 & $81,39 \%$ \\
-. Invasive Lobular Carcinoma & 6 & $13,95 \%$ \\
-. Others & 2 & $4,65 \%$ \\
Histological grading & & \\
-. Grade I & 2 & $4,65 \%$ \\
-. Grade II & 13 & $30,23 \%$ \\
-. Grade III & 28 & $65,12 \%$ \\
\hline
\end{tabular}


ISSN 25980580

Table 4. Correlation between Grading of Breast Cancer Malignancy with Basic Haematology Parameters

\begin{tabular}{lcc}
\hline & \multirow{2}{*}{ Variables } & \multicolumn{2}{c}{ Grade } \\
\cline { 2 - 3 } & $\mathbf{R}$ & $\boldsymbol{P}$ \\
\hline Hemoglobin & 0,251 & 0,104 \\
Leukocyte & 0,111 & 0,479 \\
Platelet & $-0,091$ & 0,562 \\
Neutrophil & 0,110 & 0,484 \\
Limphocyte & $-0,227$ & 0,144 \\
Monocyte & $-0,017$ & 0,915 \\
\hline
\end{tabular}

Table 5. Correlation between LMR with Grading of Breast Cancer Malignancy

\begin{tabular}{|c|c|c|c|c|c|c|c|c|c|}
\hline \multicolumn{10}{|c|}{ Breast Cancer } \\
\hline \multirow{2}{*}{ LMR } & \multicolumn{2}{|c|}{ Grade I } & \multicolumn{2}{|c|}{ Grade II } & \multicolumn{2}{|c|}{ Grade III } & \multicolumn{2}{|c|}{ Total } & \multirow{2}{*}{$\mathbf{p}$} \\
\hline & $\mathbf{n}$ & $\%$ & $\mathbf{n}$ & $\%$ & $\mathbf{n}$ & $\%$ & $\mathbf{n}$ & $\%$ & \\
\hline$<2,5$ & & & 3 & 6,98 & 11 & 25,58 & 14 & 32,56 & \\
\hline $2,5-5,0$ & 2 & 4,65 & 6 & 13,95 & 11 & 25,58 & 19 & 44,19 & 0,376 \\
\hline$>5,0$ & & & 4 & 9,3 & 6 & 13,95 & 10 & 23,26 & \\
\hline
\end{tabular}

\section{Discussion}

Recent studies shows that systemic inflammation is correlated with cancer. Inflammation impacts each step of tumorgenesis, including tumor initiation, promotion and metastatic progression. Inflammatory indicators that can be used as biomarkers in cancer prognostics are neutrophil-lymphocyte ratio, platelet-lymphocyte ratio and lymphocyte-monocyte ratio. ${ }^{9,13}$ Systemic inflammatory biomarkers are hypothesized reresenting circulating cells, specifically the lymphocytes to monocytes ratio (LMR), LMR value have been reported to be prognostic factors in breast cancer. Accumulated evidence shows that infiltration inflammatory cells in the tumor microenvironment have a significant effect on tumor initiation, proliferation, angiogenesis and metastasis. ${ }^{14}$

Lymphocytes have a major role in suppressing proliferation, migration of cancer cells, and cell death. Tumor Infiltrating Lymphocytes (TIL) is a vital component of the antitumor immunity microenvironment and is involved in several stages of tumor development. Cytotoxic lymphocytes and cytotoxic $\mathrm{T}$ cells play an important role in removing residual cancer cells. Therefore, a decrease in the value of lymphocytes affecting immunological reactions to tumors, thereby increasing tumor growth and metastasis. ${ }^{9,15}$ 
Monocytes are known to have an effect on tumor growth. Monocytes can infiltrate tumors and will differentiate into Tumor Associated Macrophages (TAMs). It is known that TAM is involved in proliferation, invasion, metastasis, tumor neovascularization and recurrence. Therefore, the number of monocytes can represent the number of TAMs. Increased monocyte count represent a high tumor burden in patients with cancer. ${ }^{9,15}$

According to mechanism of lymphocytes and monocytes, LMR is believed to reflect the host's immune status and tumor development level. Low lymphocyte with high monocyte counts represent inadequate anti-tumor immunity and increased tumor burden. A low LMR predictive value associated with a worse cancer prognosis. LMR value represent the balance between benefits of lymphocytes role with the lack of monocytes role in the process of cancer Development. ${ }^{9}$ Lymphocytes to monocytes ratio (LMR) values have also been found to be associated with survival rates of malignant lymphoma patients and various solid tumors, including breast cancer. ${ }^{16}$

Many studies have shown that LMR values are reported to be prognostic factor of several cancer, including breast cancer. He, et al reported the LMR value can be used as a predictive factor in long-term survival of triple negative breast cancer patients. ${ }^{17}$ In line with these findings, Curcio et al found that LMR can be used as a marker in breast cancer, especially in triple negative types of breast cancer and luminal B-like breast cancer. ${ }^{18}$ In addition, Hu et al reported that low LMR values associated with poor prognosis in breast cancer. ${ }^{19}$ High LMR values suggesting a good prognosis in breast cancer patients with luminal subtypes treated with 5-flourouracil chemotherapy. ${ }^{13}$

Other studies assessing the prognostic function of B cells infiltrating tumors, especially in breast cancer, report positive or negative prognostic values. Recent meta-analysis reported an increase in CD20+ B cell counts associated with disease free survival (DFS) and breast cancer specific survival (BCSS) ${ }^{20}$ In contrast with that, CD19+ B cell counts have a poor prognostic value in breast cancer patient that has been assess with indicators such as estrogen / progesterone receptors, metastasis to lymph nodes, tumor histology Grande III, and T4 status against TNM. ${ }^{21}$ In addition, some studies reports that there is a weak correlation between CD20 + cells with histological features, Cycling A or Ki67 in breast cancer. ${ }^{22}$ 
Several previous studies illustrate the correlation results in certain breast cancer subtypes showing that each subtypes has heterogeneous antitumor cells. Thus, each of these cancer subtypes will induce a different host response. Thereby, the value or level of each immunological cell produced by each subtype has a different number and the. ${ }^{13,15}$

In addition, the cut-off determination of LMR values in several studies are using various reference. Therefore, there is no standard reference in determining categories in LMR values. ${ }^{13}$ Overall, the prognostic assessment of breast cancer using lymphocytes or B cells shows various results depends on the condition of the disease. furthermore, the determination of LMR value cut-off point must be adjusted to the type or subtype of cancer, thereby different immune responses and other biases can be eliminated. ${ }^{13,15}$

\section{Conclusion}

Lymphocytes to monocytes ratio (LMR) value cannot be utilize as a parameter for evaluating prognosis of breast cancer.

\section{Acknowledgment}

We are specifically grateful to Head of Lampung University and Dean of the Faculty of Medicine of Lampung University for the assistance given. We also thank all the volunteers who responded in this study; officers and staff of the Urip Sumoharjo Hospital Bandar Lampung, for their efforts in helping us.

\section{References}

1. World Health Organization (WHO). Latest global cancer data: Cancer burden rises to 18.1 million new cases and 9.6 million cancer deaths in 2018. Genewa: WHO; 2018.

2. Breast Cancer Statistic. London: Word Cancer Research Fund. Breast Cancer Statistic: Breast cancer is the most common cancer in women worldwide, 2018. (Accessed January 10, 2020, at https://www.wcrf.org/dietandcancer/cancer-trends/breast-cancer-statistics).

3. Kementerian Kesehatan RI. Profil Kesehatan Republik Indonesia Tahun 2017. Jakarta: Kementerian Kesehatan RI; 2018.

4. Kementerian Kesahatan RI. Profil Kesehatan Republik Indonesia Tahun 2018. Jakarta: Kementerian Kesehatan RI; 2019. 
5. Yang H, Pawitan Y, He W, Eriksson L, Holowko N, Hall P, et al. Disease trajectories and mortality among women diagnosed with breast cancer. The Breast. 2019; 21(95):1-8.

6. Cao SS, Lu CT. Recent perspectives of breast cancer prognosis and predictive factors (Review). Oncol. Lett. 2016; 12(5):3674-8.

7. Oluogun WA, Adedokun KA, Oyenike MA, Adeyeba OA. Histological classification, grading, staging, and prognostic indexing of female breast cancer in an African population: A 10-year retrospective study. Int. J. Health Sci. 2019; 13(4):3-9.

8. Rakha EA, Reis-filho JS, Baehner F, Dabbs DJ, Decker T, Eusebi V, et al. Breast cancer prognostic classifi cation in the molecular era: the role of histological grade. Breast Cancer Reasearch. 2010; 12(207):1-12.

9. Goto, W., Kashiwagi, S., Asano, Y., Takada, K., Takahashi, K., Hatano, T., et al. Predictive value of lymphocyte-to-monocyte ratio in the preoperative setting for progression of patients with breast cancer. BMC Cancer. 2018; 18(1):1-9.

10. Adham, M., Kurniawan, A. N., Muhtadi, A. I., Roezin, A., Hermani, B., Gondhowiardjo, S., et al. Nasopharyngeal carcinoma in indonesia: Epidemiology, incidence, signs, and symptoms at presentation. Chin. J. Cancer. 2012; 31(4):185-96.

11. Yamagishi T, Fujimoto N, Nishi H, Miyamoto Y, Hara N, Asano M, et al. Prognostic significance of the lymphocyte-to-monocyte ratio in patients with malignant pleural mesothelioma. Lung Cancer. 2015; 90(1):111-7.

12. Chen J, Hong D, Zhai Y, Shen P. Meta-analysis of associations between neutrophil-tolymphocyte ratio and prognosis of gastric cancer. World J. Surg. Oncol. 2015; 13(1):1-9.

13. Ji H, Xuan Q, Yan C, Liu T, Nanding A, Zhang Q. The prognostic and predictive value of the lymphocyte to monocyte ratio in luminal-type breast cancer patients treated with CEF chemotherapy. Oncotarget. 2016; 7(23):34881-9.

14. Li M, Deng Q, Zhang L, He S, Rong J, Zheng F. The pretreatment lymphocyte to monocyte ratio predicts clinical outcome for patients with urological cancers: A meta-analysis. ELsevier. 2019; 215:5-11.

15. Li W, Ma G, Wu Q, Deng Y, Liu Y, Wang J. Prognostic value of lymphocyte-to-monocyte ratio among Asian lung cancer patients: A systematic review and meta-analysis. Oncotarget. 2017; 8(66):110606-13. 
16. Zhang GM, Zhu Y, Luo L, Wan FN, Zhu YP, Sun L. Preoperative lymphocyte-monocyte and platelet-lymphocyte ratios as predictors of overall survival in patients with bladder cancer undergoing radical cystectomy. Tumor Biol. 2015; 36:8537-43.

17. He J, Lv P, Yang X, Chen Y, Liu C, Qiu X. Pretreatment lymphocyte to monocyte ratio as a predictor of prognosis in patients with early-stage triple-negative breast cancer. Tumor Biol. 2016; 37(7):9037-43.

18. Gerratana, L., Basile, D., Zago, S., Vitale, M. G., Pelizzari, G., Bonotto, M., et al. Neutrophil-to-lymphocyte and lymphocyte-to-monocyte ratios in breast cancer. Eur. Soc. Med. Oncol. 2017; 28(5):43-67.

19. Hu R, Liu Q, Ma J, Zhou J, Liu G. Preoperative lymphocyte-to-monocyte ratio predicts breast cancer outcome: A meta-analysis. Clin. Chim. Acta. 2018; 484:1-6.

20. Mao Y, Qu Q, Chen X, Huang O, Wu J, Shen K. The prognostic value of tumor-infiltrating lymphocytes in breast cancer: A systematic review and meta-analysis. PLoS One. 2016; 11(4):1-13.

21. Guan H, Wan Y, Lan J, Wang Q, Wang Z, Li Y, et al. PD-L1 is a critical mediator of regulatory B cells and T cells in invasive breast cancer. Sci. Rep. 2016; 6(35651):1-10.

22. Löfdahl, B., Ahlin, C., Holmqvist, M., Holmberg, L., Zhou, W., Fjällskog, M. L., et al. Inflammatory cells in node-negative breast cancer. Acta Oncol. 2012; 51(5):680-6. 